\title{
Using subseasonal-to-seasonal (S2S) extreme rainfall forecasts for extended-range flood prediction in Australia
}

\author{
C. J. White ${ }^{1}$, S. W. Franks ${ }^{1}$, and D. McEvoy ${ }^{2}$ \\ ${ }^{1}$ School of Engineering and ICT, University of Tasmania, Hobart, Australia \\ ${ }^{2}$ Global Cities Research Institute, RMIT University, Melbourne, Australia
}

Correspondence to: C. J. White (chris.white@utas.edu.au)

Received: 17 March 2015 - Accepted: 17 March 2015 - Published: 11 June 2015

\begin{abstract}
Meteorological and hydrological centres around the world are looking at ways to improve their capacity to be able to produce and deliver skilful and reliable forecasts of high-impact extreme rainfall and flooding events on a range of prediction timescales (e.g. sub-daily, daily, multi-week, seasonal). Making improvements to extended-range rainfall and flood forecast models, assessing forecast skill and uncertainty, and exploring how to apply flood forecasts and communicate their benefits to decision-makers are significant challenges facing the forecasting and water resources management communities. This paper presents some of the latest science and initiatives from Australia on the development, application and communication of extreme rainfall and flood forecasts on the extended-range "subseasonal-to-seasonal" (S2S) forecasting timescale, with a focus on risk-based decision-making, increasing flood risk awareness and preparedness, capturing uncertainty, understanding human responses to flood forecasts and warnings, and the growing adoption of "climate services". The paper also demonstrates how forecasts of flood events across a range of prediction timescales could be beneficial to a range of sectors and society, most notably for disaster risk reduction (DRR) activities, emergency management and response, and strengthening community resilience. Extended-range S2S extreme flood forecasts, if presented as easily accessible, timely and relevant information are a valuable resource to help society better prepare for, and subsequently cope with, extreme flood events.
\end{abstract}

\section{Introduction}

Riverine flooding following heavy rainfall events is the most common form of flooding in Australia. Flooding of rivers in inland areas of central and western New South Wales and Queensland, as well as parts of Western Australia, can spread for thousands of square kilometres and may last for weeks or even months. The Bureau of Meteorology (BoM) is the lead national agency for streamflow forecasting and flood warning services in Australia, providing coverage of most major rivers and delivered through regional flood warning centres in each State and Territory in partnership with water agencies, local government authorities and emergency management responders.

Forecasting river levels and flood extent is a complex process. Designed to provide advance warning of a flood, warnings in Australia are generally derived from event-based fore- cast models that use real-time streamflow and rainfall observations to forecast floods with typical lead times from hours to a few days, depending on flood travel time (Elliott et al., 2005). Seasonal forecasts, in contrast, can be used to alert of a heightened chance of high river levels (and subsequently flooding) in the months ahead. The type of flood forecast provided depends on the quality of real-time rainfall, radar and river level data, the capability of rainfall and hydrological forecast models and the level of service required. The accuracy of forecasts varies with lead time (i.e. it is easier to forecast when the lead time is relatively short), spatial extent (e.g. catchment wide rather than specific location), the variable being forecast (e.g. rain or thunderstorm), as well as location (e.g. the mid-latitudes are easier to forecast than the tropics on seasonal timescales).

The BoM is currently implementing a "next-generation" flood forecasting system similar to that used in the UK and 
the USA to provide improved streamflow forecasts and flood warnings up to 10 days ahead. This is an initial response to the "Munro Review" (Munro, 2011) carried out to assess the BoM's capacity to respond to extreme weather and natural disaster events. These forecasts are designed to complement BoM's current Numerical Weather Prediction (NWP) rainfall forecasts and the existing flood forecasting and warning service. At the other end of the prediction timescale, long-range probabilistic seasonal streamflow forecasts (i.e. 3-month outlooks of total flow volumes, taking into account how wet or dry the catchments are at the start of the season as well as the underlying state of the climate) are provided by the BoM as part of climate and weather outlook service (see: http://www.bom.gov.au/water/ssf/). The seasonal streamflow forecasts use a statistical Bayesian Joint Probability (BJP) modelling system (Wang et al., 2009; Wang and Robertson, 2011; Robertson and Wang, 2012) developed by CSIRO under the Water Information Research and Development Alliance (WIRADA) to forecast seasonal total volumes of streamflows. Seasonal streamflow forecasts are provided operationally at around 70 sites across Australia, containing statements advising where the percentage chance of low, near-median or high streamflows may be expected at selected locations and where forecast skill is deemed to be acceptable through forecast verification. Bennett et al. (2014) recently focused on forecasting high streamflows in southeast Australia for the next 1-3 months using the BJP modelling system, finding positive forecast skill in 1-month high streamflow forecasts. However, whilst longer-range streamflow forecasts are capable of providing additional information when there is not enough data to make specific flood predictions, they do not provide information about flood class, predicted timing or frequency, severity or extent of flood (e.g. an increased chance of flooding where total streamflow volume is forecast to be high) due to longer lead times.

In addition to the longer-range streamflow forecasts, probabilistic seasonal rainfall outlooks and selected large-scale climate indices are also issued by the BoM using the global seasonal dynamical ensemble forecast model Predictive Ocean Atmosphere Model for Australia (POAMA; see: http://www.bom.gov.au/climate/ahead/). Output from seasonal dynamical forecast models such as POAMA can be utilised for operational monthly streamflow forecasts in Australia using a combination of output from seasonal forecast models, predictions from rainfall-runoff models and the BJP modelling system. Such an approach could therefore potentially extend the lead time of flood forecasts, but because seasonal rainfall forecasts are not that accurate for specific locations and timing flood forecasts based on seasonal rainfall forecasts will often be subject to significant uncertainty.

There is, therefore, a significant predictive gap in between the current short- to medium-range NWP weather forecasts and warnings (including the THORPEX Interactive Grand Global Ensemble (TIGGE) project for NWP forecasts; see: http://tigge.ecmwf.int/) and the statistical/dynamical sea- sonal streamflow and rainfall outlooks, which has largely yet to be explored for its potential for improving flood forecasts. This timescale is referred to the subseasonal-to-seasonal (S2S) or "intra-seasonal" forecasting timescale and is generally regarded as an extended-range 15- to 30-day (i.e. weeks 3 to 4) forecast lead time (Robertson et al., 2014). The S2S timescale, long seen as a "predictability desert", is still at a relatively early stage of development but is starting to gain interest across the flood forecast and other applications communities, with at least ten international weather centres now routinely issuing experimental or operational S2S forecasts (Vitart, 2014a). This provides a significant "window of opportunity" whereby dynamical S2S rainfall forecasts, such as those produced by POAMA, can start to be explored for streamflow and flood forecasting capabilities.

This paper presents the possibility of utilising S2S predictions for seamless "weather-to-climate" flood forecasting (Sect. 2) and provides information on the challenges on using and communicating flood forecasts on S2S timescales (Sect. 3). Finally, some concluding remarks are provided (Sect. 4).

\section{Subseasonal-to-seasonal (S2S) ensemble predictions - a new opportunity for flood forecasting?}

There is growing interest across the scientific, operational and applications communities in developing forecasts that fill the gap between NWP weather forecasts and the long-range seasonal outlooks (Vitart, 2014a; Robertson et al., 2014). This includes improving the skill of high streamflow forecasts on extended-range forecast horizons, including S2S lead times. The prospect of a period of prolonged wet or increased extreme rainfall frequency (e.g. high 1- or multi-day rainfall totals), for example, would lead to a likely increased chance of flooding. Forecasting rainfall on S2S lead times can help warn about heightened flood risk. A WMO WWRPWCRP project on S2S prediction is currently underway (Vitart et al., 2012; Vitart, 2014a) (see: http://s2sprediction.net/), aimed at improving forecast skill and understanding of the S2S timescale and to promote its uptake by operational centres and applications communities. The growing interest in S2S prediction has been triggered by a combination of growing demand from the applications communities, progress in medium-range forecasting over the past decades, and improvements in identifying and simulating key sources of predictability on the S2S timescale (see Sect. 3.1). A key deliverable of this project, due in early 2015 , will be a database of near real-time S2S forecasts and hindcasts produced by several operational meteorological institutions across the world, including BoM, ECMWF, NOAA and the UK Met Office (see: https://software.ecmwf.int/wiki/display/S2S/Home).

Although there are numerous efforts underway to operationalize aspects of $\mathrm{S} 2 \mathrm{~S}$ forecasts and to develop and demon- 
strate the potential value of applications-relevant information (Robertson et al., 2014), the S2S timescale is a new frontier for predictability research. While at a relatively early stage of development, it represents an opportunity for a range of different applications, including disaster risk reduction (DRR) activities and extended-range flood prediction (White, 2014). However, important modelling design issues remain to be resolved; including initialization techniques, initial conditions, model resolution, ocean-atmosphere coupling etc. Coordination between forecast producers needs to be improved before the full potential of S2S prediction can be realised (Vitart, 2014a). The WWRP-WCRP S2S project dataset will therefore provide a highly valuable repository for the applications communities to be able to evaluate the potential benefit of multi-model prediction of rainfall (and other variables) on the S2S timescale and its usability for important societal applications such as flood forecasting.

\section{Challenges using S2S predictions for flood forecasting}

\subsection{Identifying sources of forecast skill}

Accurate flood forecasting relies on reliable, real-time data about catchment wetness, observed rainfall and river water levels as well as verified rainfall forecasts and large-scale oceanic and atmospheric climate indicators (or "indices") on a range of prediction timescales. The $\mathrm{S} 2 \mathrm{~S}$ timescale is a relatively new forecasting timescale, beyond that of event-based NWP operational flood forecasting capabilities, therefore not all of the required data will be available in real-time, nor may it be as reliable. Vitart (2014b), however, show that the skill to predict rainfall has improved significantly over the past 10 years with a gain of about a week of predictive skill in the extended-range due to improved modelled soil moisture, initialization and rainfall calibration techniques. The ECMWF model, for example, displays higher rainfall forecast skill in the tropical regions than in the extratropics for forecasts beyond 10 days (i.e. into $\mathrm{S} 2 \mathrm{~S}$ lead times). This presents a major opportunity for flood forecasts on longer lead times such as S2S.

As with seasonal forecasting, S2S prediction is primarily based on large-scale climate indicators, where forecast skill is generally enhanced when identified large-scale indices are in positive or negative phases (e.g. Hudson et al., 2011; White et al., 2013). Understanding the relationship (or "teleconnection") between the index and runoff is therefore crucial. The large-scale El Niño-Southern Oscillation (ENSO) index is regarded as the primary driver of predictable interannual variability of rainfall across Australia (e.g. Risbey et al., 2009), with dynamical models such as POAMA used to predict the development of the La Niña (negative ENSO) events that are often associated with heavy rains across parts of Australia. Several studies have identified teleconnections between ENSO and runoff (e.g. Kiem et al.,
2003; Verdon et al., 2004; Schepen et al., 2012a) and rainfall (Hudson et al., 2011; Marshall et al., 2013). The influence of the Indian Ocean Dipole (IOD) has also been linked to rainfall in some regions of Australia (Verdon and Franks, 2005; Schepen et al., 2012a). Other climate indices have also been linked to seasonal rainfall (e.g. Langford et al., 2011; Lim et al., 2011), including the Southern Annular Mode (SAM) and the Madden-Julian Oscillation (MJO). Hudson et al. (2011) found that the forecast skill of rainfall on intraseasonal timescales was found to be increased during strong phases of the ENSO and the IOD, indicating that these slow variations of boundary forcing should be considered a source of intraseasonal climate predictability. Some of these have been used to develop skilful probabilistic forecasts of total seasonal streamflows (Wang et al., 2009; Piechota et al., 1998; Sharma, 2000) but there has, to date, been limited research on teleconnections to runoff on the extended-range timescales or to extreme rainfall on either the seasonal or S2S lead times in Australia. Bennett et al. (2014), however, show that including lagged climate indices as predictors adds little skill to 1-month high streamflow forecasts based on the BJP modelling system, but that catchment wetness is by far the most important source of predictability where catchments have longer (i.e. month-to-month) flood memory. How this translates to the S2S timescale is, as yet, unknown and is an identified subject for further research. There is also a need for improved and unified forecast quality assessment methodology (i.e. forecast verification) (Vitart, 2014a). Verification is a critical component of making forecasts useful to applications, with seamless verification important for both the S2S timescale and for extremes. White et al. (2013) show the Symmetric Extremal Dependence Index (SEDI) skill score to be an appropriate verification methodology for extremes forecasts on the S2S timescale for example.

\subsection{Merging S2S rainfall forecasts and hydrological forecasts}

The modelling approach offered by dynamical models such as POAMA combined with the enhanced understanding of the teleconnections between large-scale oceanic and atmospheric indices and rainfall-runoff presents increased sources of predictability for streamflow forecasting on the S2S timescale by applying rainfall predictions from global dynamical models to hydrological models in order to produce streamflow and flood predictions. However, S2S rainfall forecasts are not highly accurate and are generally expressed only in probabilistic terms (an example of an experimental rainfall forecast using POAMA on the S2S timescale is shown in Fig. 1). How to integrate extended-range probabilistic rainfall forecasts with existing hydrological modelling frameworks i.e. addressing issues such as model selection and calibration, observed catchment wetness, lagged and real-time forecasts, uncertainties around extremes, bias correction, requirements for high-resolution rainfall forecasts, 


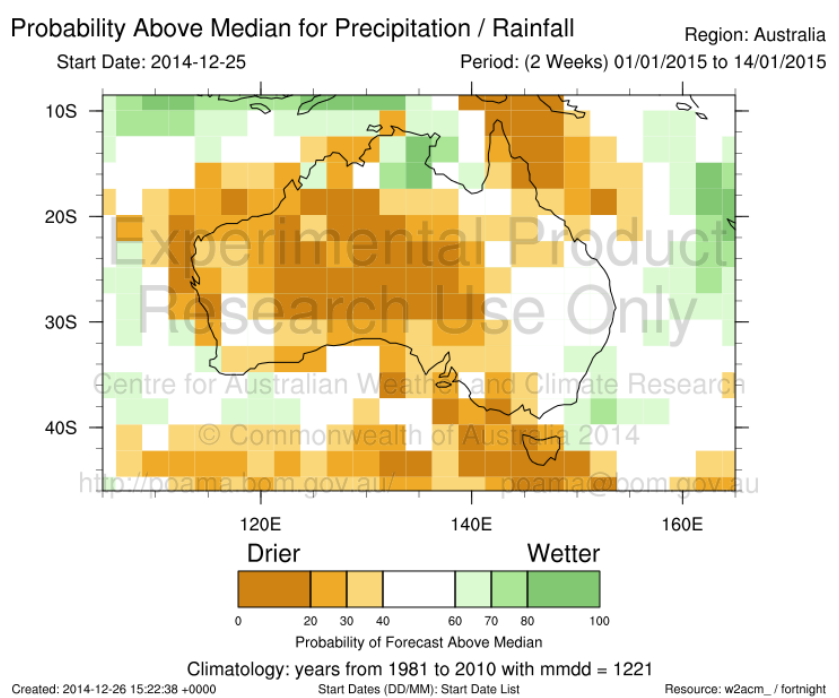

Figure 1. Example of an experimental POAMA subseasonal rainfall forecast for weeks 2 and 3 combined, showing probability of rainfall above median (obtained from the Bureau of Meteorology POAMA website: http://poama.bom.gov.au/).

integration with communication and response systems - is an active area of research in Australia (e.g. Cuo et al., 2011; Tuteja et al., 2011; Wang et al., 2012; Schepen et al., 2012b).

\subsection{Communicating flood risk and uncertainty on the S2S timescale}

Weather-related hazards, such as extended periods of flooding and drought, account for a significant proportion of natural disaster losses in Australia. While many end-users have benefited by applying weather and climate forecasts in their decision-making, "gaps" in forecasting capabilities remain, including for example at the S2S scale, and by a lack of understanding and appreciation of the complex processes and numerous facets involved in decision-making (Vitart, 2014a).

S2S predictions offer an exciting, previously unexplored forecasting timescale for flood prediction. Vitart (2014a) note that considerable returns from investment in climate science and model development are to be expected if the science of S2S prediction can be successfully connected to societal applications. However, the S2S timescale presents a significant challenge for both operational forecast centres and societal decision-makers. All forecasts, irrespective of timescale, contain uncertainty due to a range of factors. How to achieve reliable and skilful flood forecasts whilst maintaining and communicating both forecast skill and uncertainty to a range of decision-makers and response systems is a considerable challenge.

Weather and climate span a continuum of timescales with different lead times relevant to a range of end-user requirements. In the context of disaster preparedness the Red Cross/Red Crescent Climate Centre and IRI have proposed a "Ready-Set-Go!" climate services concept for making use of forecasts from the seasonal down to weather timescales based on a seamless "weather-to-climate" prediction approach (Goddard et al., 2014). This concept could be used to frame flood forecasting, whereby seasonal forecasts could be the "Ready" monitoring phase including large-scale climate indices, catchment wetness, observed rainfall events, updating contingency plans, and enabling early warning systems; sub-monthly (S2S) forecasts can then be the "Set" phase where they are used to alert volunteers, warn communities and decision-makers about an increased risk of flooding; and NWP weather forecasts and warnings would provide the "Go!" phase, used to activate volunteers, distribute instructions to communities, and evacuate if needed. In this approach, extended flood warning (on S2S lead times), response and recovery from flood hazards may be regarded as an intuitive and integral forecasting approach, illustrating the potential benefit of a seamless extreme events prediction for decision-makers. An often overlooked but valuable component of communicating forecasts successfully are the social sciences, where the use and value of weather forecast information for societal impacts is assessed on various forecasting lead times (e.g. Morss et al., 2008; Demuth et al., 2013). These approaches could be used to develop user information on flood risk, risk perception and forecast uncertainty on the S2S timescale to help improve decision-makers use weather information more effectively.

\section{Conclusions}

Skilful and reliable forecasts of flood risk beyond NWP forecasts and up to a month in advance (e.g. the shortest of the climate outlooks) have the potential to improve the management of floods. This S2S timescale is a notable gap in current flood forecast capabilities that could be utilised to help achieve seamless flood and streamflow predictions, both in Australia and internationally. The development of modelling approaches and assessing the utility of S2S forecasts has the potential to extend the range of flood forecasting and improve the skill of hydrological prediction modelling in Australia. While S2S forecasts are not as precise as short-range forecasts, forewarning of conditions that could result in large or frequent flooding in the next 2-4 weeks (or more) could allow emergency services to better plan and prepare for the impacts of floods in line with the National Strategy for Disaster Resilience that advocates greater emphasis on planning rather than the traditional reliance on response and recovery (Council of Australian Governments, 2011). Indeed, S2S predictions could actively support local resilience building by informing volunteer emergency services personnel of heightened flood risk or by ensuring people can make decisions to protect their lives and homes, as well as community infrastructure. Reliable S2S streamflow forecasts - if complementing BoM's seasonal rainfall and temperature outlook 
services - could also be used to influence and improve water management decision-making capabilities.

It is likely that predictive skill on the S2S timescale will be higher within certain "windows of opportunity" (e.g. Hudson et al., 2011), but exactly what these are or how to anticipate them is still unclear (Vitart, 2014a). Relevant science questions - ranging from sources of predictability, forecasting systems design, forecast verification, to the final integration of these forecasts in user applications and response systems - are all issues that need to be addressed before they can be integrated in extended-range flood predictions. The new S2S database may also hold some limitations, such as the $1.5^{\circ}$ archiving resolution, the choice of variables available, as well as the unknown potential of multi-model ensembles to improve flood forecasts. Similarly, how to effectively communicate flood risk to a range of decision-makers using forecasts on extended-range timescales that contain a high degree of uncertainty remains a significant challenge. The weather and climate research communities will need to collaborate with end-users to co-develop new focused warnings and forecasts that meet the needs of decision-makers. Notwithstanding the challenges, as flood forecasts improve in accuracy and are integrated with communication and response systems over longer lead times, they can become highly valuable resources for disaster risk reduction and adaptation planning.

Acknowledgements. The authors would like to thank A. W. Robertson (IRI) and F. Vitart (ECMWF) for their insightful comments and advice in the preparation of this manuscript.

\section{References}

Bennett, J. C., Wang, Q. J., Pokhrel, P., and Robertson, D. E.: The challenge of forecasting high streamflows $1-3$ months in advance with lagged climate indices in southeast Australia, Nat. Hazards Earth Syst. Sci., 14, 219-233, doi:10.5194/nhess-14-219-2014, 2014.

Council of Australian Governments: National Strategy for Disaster Resilience, Council of Australian Governments, Canberra, 2011.

Cuo, L., Pagano, T. C., and Wang, Q. J.: A review of quantitative precipitation forecasts and their use in short-to medium range streamflow forecasting, J. Hydrometeorol., 12, 713-728, doi:10.1175/2011JHM1347.1, 2011.

Demuth, J. L., Morss, R. E., Lazo, J. K., and Hilderbrand, D. C.: Improving effectiveness of weather risk communication on the NWS Point-and-Click web page, Weather Forecast., 28, 711726, 2013.

Elliott, J., Catchlove, R., Sooriyakumaran, S., and Thompson, R.: Recent advances in the development of flood forecasting and warning services in Australia, International Conference on Innovation, Advances and Implementation of Flood Forecasting Technology, Tromsø, Norway, 2005, 17-19 October, 2005.

Goddard, L., Baethgen, W. E., Bhojwani, H., and Robertson, A. W.: The International Research Institute for Climate \& Society: why, what and how, Earth Perspect., 1-10, 2014.
Hudson, D., Alves, O., Hendon, H. H., and Marshall, A. G.: Bridging the gap between weather and seasonal forecasting: intraseasonal forecasting for Australia, Q. J. Roy. Meteorol. Soc., 137, 673-689, 2011.

Kiem, A. S., Franks, S. W., and Kuczera, G.: Multi-decadal variability of flood risk, Geophys. Res. Lett., 30, 1035, doi:10.1029/2002GL015992, 2003.

Langford, S., Hendon, H. H., and Lim, E-P.: Assessment of POAMA's predictions of some climate indices for use as predictors of Australian rainfall, CAWCR Technical Report No. 031, 2011.

Lim, E-P., Hendon, H. H., Anderson, D. L. T., Charles, A., and Alves, O.: Dynamical, statistical-dynamical and multi-model ensemble forecasts of Australian spring season rainfall, Mon. Weather Rev., 139, 958-975, 2011.

Marshall, A. G., Hudson, D., Hendon, H. H., Pook, M., Alves, O., and Wheeler M. C.: Simulation and prediction of blocking in the Australian region and its influence on intra-seasonal rainfall in POAMA-2, Clim. Dynam., 43, 1915-1937, doi:10.1007/s00382013-1974-7, 2013.

Morss, R. E., Demuth, J. L., and Lazo, J. K.: Communicating Uncertainty in Weather Forecasts: A Survey of the U.S. Public, Weather Forecast., 23, 974-991, 2008.

Munro, C.: Review of the Bureau of Meteorology's capacity to respond to future extreme weather and natural disaster events and to provide seasonal forecasting services, Department of the Environment, available at: http://www.environment.gov.au/resource (last access: 12 January 2015), 2011.

Piechota, T. C., Chiew, F. H. S., Dracup, J. A., and McMahon, T. A.: Seasonal streamflow forecasting in eastern Australia and the El Niño-Southern Oscillation, Water Resour. Res., 34, 3035-3044, doi:10.1029/98WR02406, 1998.

Risbey, J. S., Pook, M. J., McIntosh, P. C., Wheeler, M. C., and Hendon, H. H.: On the remote drivers of rainfall variability in Australia, Mon. Weather Rev., 137, 3233-3253, 2009.

Robertson, A. W., Kumar, A., Peña, M., and Vitart, F.: Improving and Promoting Sub-seasonal to Seasonal Prediction, B. Am. Meteorol. Soc., doi:10.1175/BAMS-D-14-00139.1, in press, 2014.

Robertson, D. E. and Wang, Q. J.: A Bayesian approach to predictor selection for seasonal streamflow forecasting, J. Hydrometeorol., 13, 155-171, doi:10.1175/JHM-D-10-05009.1, 2012.

Schepen, A., Wang, Q. J., and Robertson, D. E.: Evidence for using lagged climate indices to forecast Australian seasonal rainfall, J. Climate, 25, 1230-1246, doi:10.1175/JCLI-D-1100156.1, 2012a.

Schepen, A., Wang, Q. J., and Robertson, D. E.: Combining the strengths of statistical and dynamical modeling approaches for forecasting Australian seasonal rainfall, J. Geophys. Res., 117, D20107, doi:10.1029/2012JD018011, 2012b.

Sharma, A.: Seasonal to interannual rainfall probabilistic forecasts for improved water supply management: part 3 - a nonparametric probabilistic forecast model, J. Hydrol., 239, 249-258, doi:10.1016/S0022-1694(00)00348-6, 2000.

Tuteja, N. K., Shin, D., Laugesen, R., Khan, U., Shao, Q., Wang, E., Li, M., Zheng, H., Kuczera, G., Kavetski, D., Evin, G., Thyer, M., MacDonald, A., Chia, T., and Le, B.: Experimental evaluation of the dynamic seasonal streamflow forecasting approach, Technical Report, Bureau of Meteorology, Melbourne, Australia, 2011. 
Verdon, D. C. and Franks, S. W.: Indian Ocean sea surface temperature variability and winter rainfall: Eastern Australia, Water Resour. Res., 41, W09413, doi:10.1029/2004WR003845, 2005.

Verdon, D. C., Wyatt, A. M., Kiem, A. S., and Franks, S. W.: Multidecadal variability of rainfall and streamflow: Eastern Australia, Water Resour. Res., 40, W10201, doi:10.1029/2004WR003234, 2004.

Vitart, F.: Sub-Seasonal to Seasonal Prediction: linking weather and climate, Proc. of the World Weather Open Science Conference (WWOSC), Montreal, Canada, 16-21 August, 2014a.

Vitart, F.: Evolution of ECMWF sub-seasonal forecast skill scores, Q. J. Roy Meteorol. Soc., 140, 1889-1899, 2014b.

Vitart, F., Robertson, A. W., and Anderson, D. L. T.: Subseasonal to seasonal prediction project: Bridging the gap between weather and climate, WMO Bull., 61, 23-28, 2012.

Wang, Q. J. and Robertson, D. E.: Multisite probabilistic forecasting of seasonal flows for streams with zero value occurrences, Water Resour. Res., 47, W02546, doi:10.1029/2010WR009333, 2011.
Wang, Q. J., Robertson, D. E., and Chiew, F. H. S.: A Bayesian joint probability modeling approach for seasonal forecasting of streamflows at multiple sites, Water Resour. Res., 45, W05407, doi:10.1029/2008WR007355, 2009.

Wang, Q. J., Schepen, A., and Robertson, D. E.: Merging seasonal rainfall forecasts from multiple statistical models through Bayesian model averaging, J. Climate, 25, 5524-5537, doi:10.1175/JCLI-D-11-00386.1, 2012.

White, C. J.: The 2013 Churchill Fellowship to investigate longrange forecasting of extreme weather events and to explore improved methods of communication for disaster risk reduction for Australia, University of Tasmania, available at: http://ecite.utas. edu.au/97440 (last access: 12 January 2015), 2014.

White, C. J., Hudson, D., and Alves, O.: ENSO, the IOD and intraseasonal prediction of heat extremes across Australia using POAMA-2, Clim. Dynam., 43, 1791-1810, doi:10.1007/s00382013-2007-2, 2013. 